\title{
Erratum to: Avoidable binary patterns in partial words
}

\author{
F. Blanchet-Sadri - Robert Mercaş - Sean Simmons • \\ Eric Weissenstein
}

Published online: 8 December 2011

(C) Springer-Verlag 2011

\section{Erratum to: Acta Informatica (2011) 48:25-41 DOI 10.1007/s00236-010-0129-0}

In the paper "F. Blanchet-Sadri, R. Mercaş, S. Simmons and E. Weissenstein, Avoidable binary patterns in partial words, Acta Informatica 48(1) (2011) 25-41", the first sentence of Section 5 as well as Theorem 6 should be deleted. The error is that overlap-freeness does not necessarily coincide with the pattern $\alpha \beta \alpha \beta \alpha$ being avoidable. It coincides only in the case when the considered overlaps have length greater than three. Thus, an infinite word might contain factors of the form aaa while still avoiding the pattern $\alpha \beta \alpha \beta \alpha$, where $a$ is a letter of the alphabet the word is defined on. Thus, given the fact that the pattern $\alpha \beta \alpha \beta \alpha$ is 3 -avoidable and, obviously, this pattern is not 1-avoidable, it remains open the question of whether $\alpha \beta \alpha \beta \alpha$ is 2-avoidable in partial words. Consequently, the pattern $\alpha \beta \alpha \beta \alpha$ should be removed from Statement 2 of Theorem 10 and a new statement should be added stating that:

The online version of the original article can be found under doi:10.1007/s00236-010-0129-0.

F. Blanchet-Sadri $(\bowtie)$

Department of Computer Science, University of North Carolina, P.O. Box 26170, Greensboro, NC 27402-6170, USA

e-mail: blanchet@uncg.edu

R. Mercaş

GRLMC, Departament de Filologies Romàniques, Universitat Rovira i Virgili,

Av. Catalunya 35, Tarragona 43002, Spain

e-mail: robertmercas@gmail.com

S. Simmons

Department of Mathematics, The University of Texas at Austin,

2515 Speedway Rm 8, Austin, TX 78712-0233, USA

E. Weissenstein

Department of Mathematical Sciences, Rensselaer Polytechnic Institute,

Amos Eaton 301, 110 8th Street, Troy, NY 12180, USA 
"The binary pattern $\alpha \beta \alpha \beta \alpha$ has avoidability index 2 or 3." In conclusion, the paper states two open problems, namely, whether the pattern $\alpha \beta \alpha \beta \alpha$ has avoidability index 2 as in the case of full words, and whether the pattern $\alpha \beta \beta \alpha$ has avoidability index 3 . 